\title{
Diffusive turn-off transients in current modulated multitransverse mode VCSELs
}

\author{
Angel Valle ${ }^{* a}$, Josep Mulet ${ }^{b}$, Luis Pesquera ${ }^{a}$ and Salvador Balle ${ }^{b}$ \\ ${ }^{a}$ Instituto de Física de Cantabria (IFCA) \\ (Consejo Superior de Investigaciones Científicas-Universidad de Cantabria) \\ Facultad de Ciencias, Avda. Los Castros s/n \\ E-39005 Santander Spain. \\ ${ }^{b}$ Instituto Mediterráneo de Estudios Avanzados (IMEDEA) \\ (Consejo Superior de Investigaciones Científicas-Universitat de les Illes Balears) \\ Campus Universitat Illes Balears \\ E-07071 Palma de Mallorca Spain.
}

\begin{abstract}
Secondary pulsations are an example of diffusive turn-off transients that can limit the performance of VCSELs in optical communication systems. Secondary pulsations are firstly analysed by using a model where a modal expansion of the electric field is performed. The maximum power of the secondary pulsations and the time at which they appear fluctuate when the spontaneous emission noise is present. A linear relation between the two previous quantities for each individual turn-off event is found. In the single-mode regime, the averaged maximum power during turn-off transients increases when increasing the injection current. However, in the multi-mode regime, the strength of secondary pulsations decreases when increasing the current. Secondary pulsations are also analysed by using a spatio-temporal description of the VCSEL dynamics, where the modal profiles are determined from the distribution of injected carriers and the thermal lens. This model also incorporates polarization effects and a frequency-dependent susceptibility. In this model, the carrier-induced refractive index changes increase the strength of secondary pulsations as compared to that obtained with the modal expansion. It is also shown that the use of ring-shaped electrical contact enhances the strength of secondary pulsations, while it decreases when multi-transverse mode operation is present.
\end{abstract}

Keywords: Semiconductor Lasers, Vertical cavity lasers, Transverse modes, Current modulation.

\section{INTRODUCTION}

Vertical-cavity surface-emitting lasers (VCSELs) are key components in the fast-growing market for short haul, multi-gigabit speed optical communication systems. In these applications the VCSEL is modulated by a highfrequency digital bit stream. Evaluation of eye diagrams for these systems indicates that several irregularities like mode jumps, relaxation oscillations, jitter and turn-off transients can limit the use of the VCSEL to frequencies significantly lower than the $3 \mathrm{~dB}$ point. ${ }^{1}$ One of these irregularities, the turn-off transients, consists in the generation of significant optical power during the turn-off of the VCSEL. This power can appear as secondary pulsations, bumps or slow optical tails. Secondary pulsations were theoretically predicted in single transverse mode VCSELs ${ }^{2}$ and subsequently observed in experiments. ${ }^{3-6}$ Secondary pulsations are thought to be caused by the joint effect of two spatial mechanisms: lateral carrier diffusion and Spatial Hole Burning (SHB), ${ }^{2}$ that is the mechanism leading to multitransverse mode operation of VCSELs ${ }^{7,8}$. Preliminar studies indicate that multitransverse mode operation significatively decreases the strength of the secondary pulsations. ${ }^{9}$

In this paper we extend the analysis of turn-off transients in single-mode index-guided VCSELs performed in Ref. [2] to the multi-transverse-mode case. This extension is accomplished by using two different approaches, with increasing levels of complexity. Secondary pulsations are firstly analysed by using a model where the

* Other author information: (Send correspondence to A.V.)

A.V: Email: valle@ifca.unican.es; Telephone: 34-942-201465; Fax: 34-942-200935. 
electric field is expanded in terms of transverse modes fixed by a built-in waveguide. Secondary pulsations are characterised by the maximum power during the turn-off and by the time at which that maximum appears. Both quantities fluctuate when the spontaneous emission noise is taken into account. This model allows us to study the statistics of these quantities. A linear relation between the maximum power and the time at which it appears for each individual turn-off event is found. The strength of the secondary pulsations is shown to be dependent on the transverse mode character of the VCSEL. In the single-mode regime, averaged maximum power during turn-off increases when increasing the injection current during turn-on. However, in the multi-mode regime, the strength of the secondary pulsations decreases when increasing the current. Secondary pulsations are also analysed by using a more complete spatio-temporal model, where the modal profiles are determined from the distribution of injected carriers and by the thermal lensing effects. This model also incorporates polarization effects and a frequency-dependent susceptibility of the active material. It is shown that the secondary pulsations are stronger than those obtained with the former model due to the carrier-induced refractive-index changes. It is also shown that the use of ring-shaped electrical contacts increases the strength of the secondary pulsations. Results obtained from this detailed model also indicate that the strength of the secondary pulsations decreases when considering multi-transverse mode operation.

The paper is organised as follows. In section 2 the modal expansion model is presented. This model is used to study the impact of spontaneous emission noise and multimode operation on the strength of secondary pulsations. Section 3 is devoted to present the results obtained from the numerical simulation of the spatiotemporal model. The impact of thermal lensing and multimode operation on the strength of secondary pulsations are also described. Finally, section 4 is devoted to summarise and discuss our results.

\section{MODAL EXPANSION MODEL}

The model utilised in this section incorporates both spatial dependence of carrier and optical field profiles. The model can be used to describe dynamics in VCSELs provided that the modal profiles are determined by an effective waveguide structure. The model describes a cylindrical weakly-index guided VCSEL as illustrated in Fig. 1 of Ref. [2]. The refractive index is slightly greater in the core region than in the cladding region. The appropriate modes for the assumed VCSEL structure are conventionally denoted as the $L P_{m n}$ modes, where $2 m(n-1)$ stands for the number of field nodes in the azimuthal (radial) direction. We consider a $\phi_{g}=5 \mu m$ waveguide diameter, and a $\Delta n=0.007$ index step waveguide. For this structure, the modes supported are $L P_{01}$, $L P_{02}, L P_{11}$, and $L P_{21}$. Assuming that the VCSEL can operate in several transverse modes simultaneously the electric field is expressed as

$$
E(r, t)=\frac{1}{2} \sum_{m n} \psi_{m n}(r) E_{m n}(t) e^{-i \omega_{m n} t}+c c .
$$

where $E_{m n}(t), \psi_{m n}(r)$ and $\omega_{m n}$ are, respectively, the complex field amplitude, electrical field profile and frequency of the $L P_{m n}$ transverse mode, while $r$ is the modulus of the spatial vector in the active layer. In this simple model we have assumed azimuthally independent modal field profiles with the same polarization direction for all transverse modes.

The active region of the device is taken to be of width $d=25 \mathrm{~nm}$, and the injected current density, $j(r)$, is uniformly distributed over a disk of diameter $\phi_{c}=6 \mu m\left(j(r)=j\right.$ if $r<\phi_{c} / 2$, and $j(r)=0$ elsewhere). The complex amplitude of each transverse mode is governed by a rate equation that reads

$$
\frac{d E_{m n}(t)}{d t}=\frac{1-i \alpha}{2}\left[v_{g} \Gamma g_{m n}(t)-\kappa_{m n}\right] E_{m n}(t)+\sqrt{\beta \pi A d \int N(r, t) r d r \xi_{m n}(t)},
$$

where $g_{m n}(t)$ and $\kappa_{m n}$ are the modal gain and losses, respectively, of the $L P_{m n}$ mode, $\beta$ is the spontaneous emission factor, $A$ is the nonradiative recombination rate, $v_{g}$ is the group velocity, $\alpha$ is the linewidth enhancement factor $(\alpha=3)$, and $\xi_{m n}(t)$ is a complex Gaussian noise term of zero mean and time correlation given by 
$\left\langle\xi_{m n}(t) \xi_{m^{\prime} n^{\prime}}^{*}(s)\right\rangle=2 \delta_{m m^{\prime}} \delta_{n n^{\prime}} \delta(t-s)$. The numerical value and meaning of the other parameters can be found in Table 1. The modal gain of the $L P_{m n}$ mode is given by

$$
g_{m n}(t)=\frac{\int_{0}^{\infty} \psi_{m n}^{2}(r) A_{0}\left(N(r, t)-N_{t}\right) r d r}{\overline{\psi_{m n}^{2}}},
$$

where $N(r, t)$ is the carrier density, $N_{t}$ is the carrier density at transparency, $A_{0}$ is the gain coefficient, $\psi_{m n}(r)$ is electric field profile of the $L P_{m n}$ mode, and $\overline{\psi_{m n}^{2}}=\int_{0}^{\infty} \psi_{m n}^{2}(r) r d r$. The modal gain then represents the degree of spatial overlapping between the mode intensity profile and the carrier distribution. Modal gain is the dominant mechanism for modal selection since the assumed index step is small. ${ }^{10}$

In order to determine the temporal evolution of the modal gain and power it is necessary to calculate the time dependence of the spatial distribution of the carrier density in the laser active region

$$
\frac{\partial N(r, t)}{\partial t}=\frac{\mathcal{D}}{r} \frac{\partial}{\partial r}\left(r \frac{\partial N(r, t)}{\partial r}\right)-A N(r, t)+\frac{j(r)}{e d}-\sum_{m n} a_{m n} \psi_{m n}^{2}(r) A_{0}\left(N(r, t)-N_{t}\right)\left|E_{m n}(t)\right|^{2}
$$

where $a_{m n}=v_{g} \Gamma /\left(2 \pi d \overline{\psi_{m n}^{2}}\right)$ and $\mathcal{D}$ is the diffusion constant. The evolution of the electric field is found by integrating rate equations for the complex modal amplitudes and the continuity equation for the carrier profile. A more complete description of the model together with full details of numerical integration can be found elsewhere. ${ }^{11,12}$

The strength of the secondary pulsations is found to be dependent on the transverse mode character of the VCSEL. ${ }^{9}$ The study of this dependence is easier in VCSELs that may exhibit single or multitransverse mode behaviour depending on the current injection. This occurs, for instance, in surface relief VCSELs, designed to operate in $L P_{01}$ mode for a wide current range. ${ }^{13}$ We model these VCSELs by assuming that higher-order transverse modes experience higher modal losses $\left(\kappa_{m n}=400 n s^{-1}\right)$ than the $L P_{01} \operatorname{mode}^{13}\left(\kappa_{01}=300 n s^{-1}\right)$. In this way the VCSEL operates in the fundamental mode, $L P_{01}$, for current densities below $4.5 j_{t h}$, where $j_{t h}=1.53 \mathrm{kA} / \mathrm{cm}^{2}$ is the threshold current density. Above that value the laser becomes multimode due to SHB effects. ${ }^{12}$ In fact, the transverse mode resolved L-I curve indicates that almost all optical power is delivered

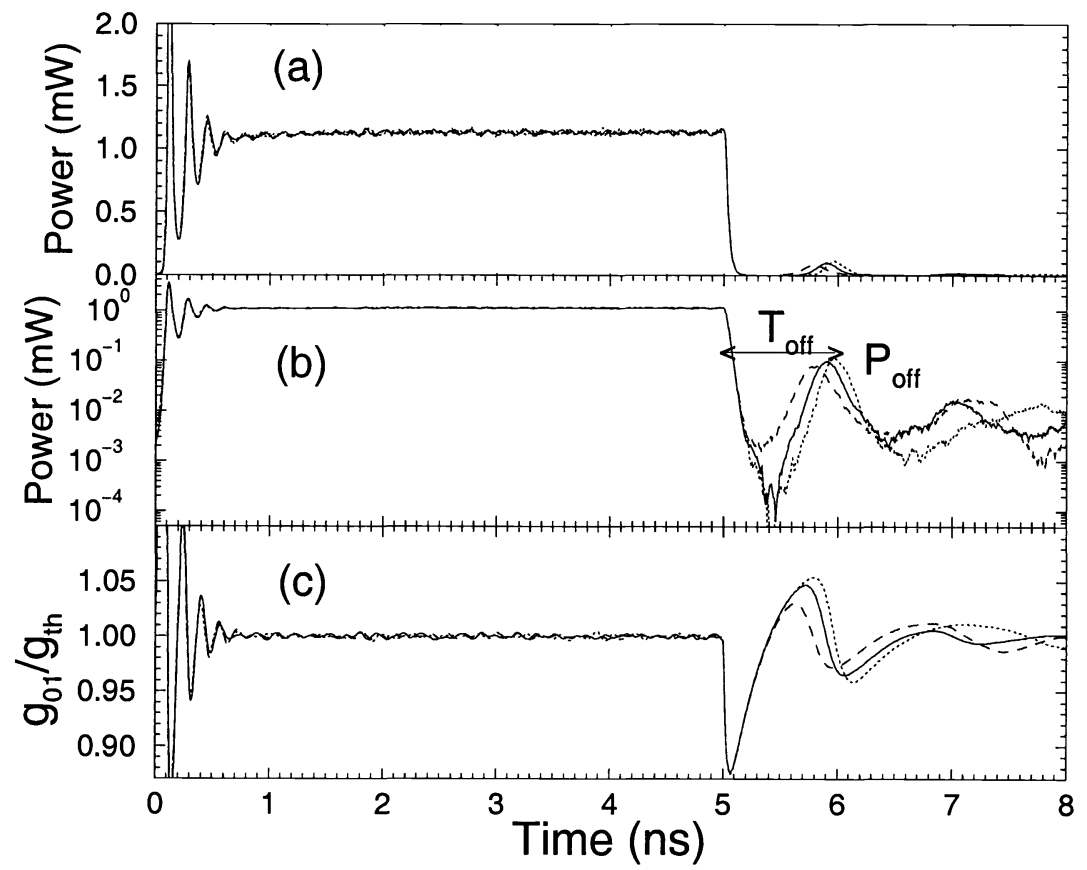

Figure 1. Temporal evolution of (a)-(b) laser optical power and (c) modal gain for three different switching events, when $j_{o n}=4 j_{t h}$. Secondary pulsations appear with $O F F / O N \sim 10 \%$ and $T_{o f f} \sim 900 p s$. 
in $L P_{01}$ and $L P_{21}$ modes when $j>4.5 j_{t h}$. First, let us consider the dynamical evolution of the VCSEL in the single-mode regime, when it is subject to a square-wave modulation current. The laser is turned-on with a current density $j=j_{o n}=4 j_{t h}$ for 5 ns and then turned-off with $j=j_{b i a s}=j_{t h}$. The dynamical evolution for three different switching events has been superimposed in Fig. 1. In this figure we can see that, after the laser is turned-off, there is emission of optical pulses containing significant optical power.

The origin of these secondary pulsations is due to a spatial redistribution of carriers within the active region. ${ }^{2}$ While the laser is driven above threshold, quite a deep hole is burned in the carrier density due to stimulated recombination caused by emission in the $L P_{01}$ mode. Following the laser turn-off, the carrier density distribution becomes more uniform because the source of the hole burning is removed and carrier diffusion processes become relevant. Since the modal gain depends on the spatial overlap between the modal profile and the carrier distribution, the process of spatial redistribution of carriers produces a recovery of the modal gain [see Fig. 1(c)], that finally leads to the emission of a secondary pulse. We note that secondary pulsations during the turn-off also occur when $j_{\text {bias }}$ is below threshold and then, these phenomena can not be predicted from simple rate equations in which spatial effects are disregarded. Secondary pulsations can be characterised by the maximum power achieved during the turn-off transient, $P_{o f f}$, and by the time at which that maximum appears, $T_{o f f}$. Both quantities have been indicated in Fig. 1 (b). The effect of spontaneous emission noise during the VCSEL turn-off can also be appreciated in that figure. Noise is important during the decay of the VCSEL power, when the laser intensity is small. Noise induces random fluctuations in the quantities $P_{o f f}$ and $T_{o f f}$. The simplicity of the model used in this section allows us to simulate a rather large number of modulation periods in a reasonable amount of computing time. $10^{4}$ periods have been simulated to obtain the probability density functions of $P_{\text {off }}$ and $T_{\text {off }}$, that are shown in Fig. 2. Both density functions, p.d.f $\left(P_{\text {off }}\right)$ and p.d.f $\left(T_{o f f}\right)$, display a slowly decaying tail for large values that extend to $0.14 \mathrm{~mW}$ and $1300 \mathrm{ps}$, respectively. Another quantity that becomes random due to spontaneous emission noise is the time at which the power of the secondary pulsation reaches a fixed level during the VCSEL turn-off, $\tau$. The probability density function of this quantity, $P(\tau)$, when the decision level is fixed at $0.01 \mathrm{~mW}$ is also plotted in Fig. 2(b).
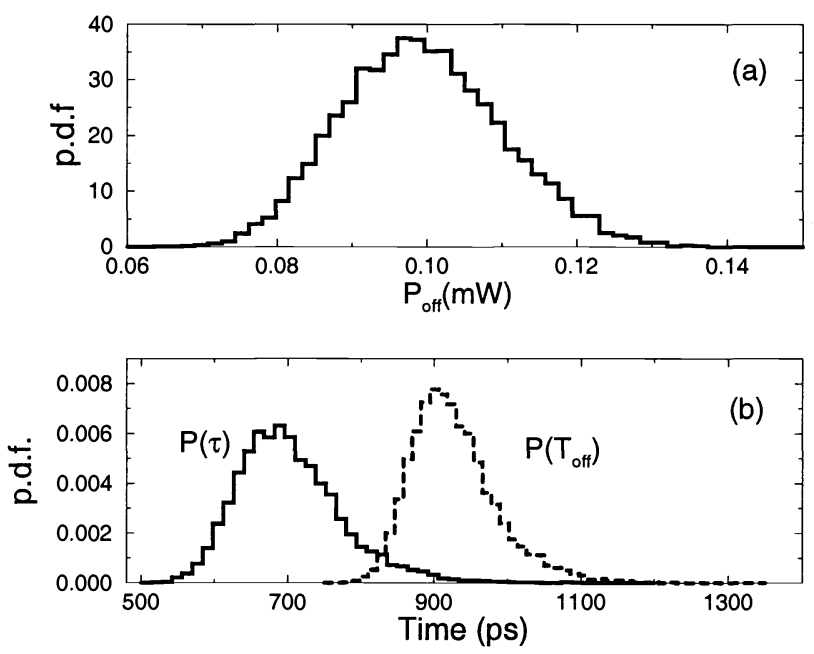

Figure 2. Probability density functions of (a) the maximum power during the turn-off, $P_{\text {off }}$, (b) time at which the maximum intensity during turn-off is reached, $T_{\text {off }}$ (dashed line) and time at which the secondary pulsation reaches a fixed power level during turn-off, $\tau$ (solid line). Parameters: $j_{o n}=4 j_{t h}$ and $j_{b i a s}=j_{t h}$.

A relationship between the previous random quantities can be infered from Fig. 1. In this figure, it can be seen that the secondary pulsations with greater power are those that appear later. This is confirmed when plotting $P_{o f f}$ vs $T_{o f f}$, and $P_{o f f}$ vs $\tau$, for all switching-off events. In fact, we find that both previous relationships are approximately linear, as it can be seen in Fig. 3. Similar linear relations, between the maximum power during turn-on and the time at which the intensity reaches a fixed value, were found when gain-switching a single mode semiconductor laser. ${ }^{14}$ Linear correlation is poor for small values of the injected current, as it can be seen 
in Figs. 3 (a)-(b). The correlation improves when increasing the current, while maintaining the single-mode operation [see Figs. 3 (c)-(d)]. The correlation again decreases when the current is increased in such a way that the VCSEL enters in the multi-mode regime [see Figs. 3 (e)-(f)]. Comparison between Figs. 3(d) and 3(f) indicates that the change from the single to multi-mode regime makes the secondary pulsations to appear later and to become weaker. These results suggest a qualitative dependence of the secondary pulsation phenomenon on the modal characteristics of the VCSEL.

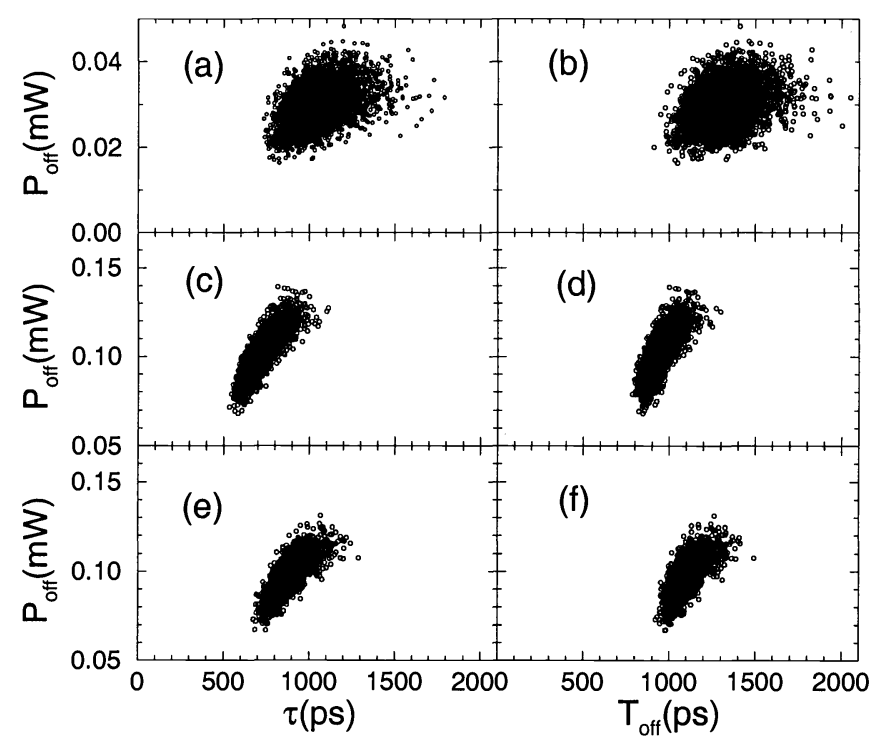

Figure 3. $P_{o f f}$ vs $\tau$ for (a) $j_{o n}=2 j_{t h}$, (c) $j_{o n}=4 j_{t h}$ and (e) $j_{o n}=8 j_{t h}$. $P_{o f f}$ vs $T_{o f f}$ for (b) $j_{o n}=2 j_{t h}$, (d) $j_{o n}=4 j_{t h}$ and (f) $j_{o n}=8 j_{t h}$.
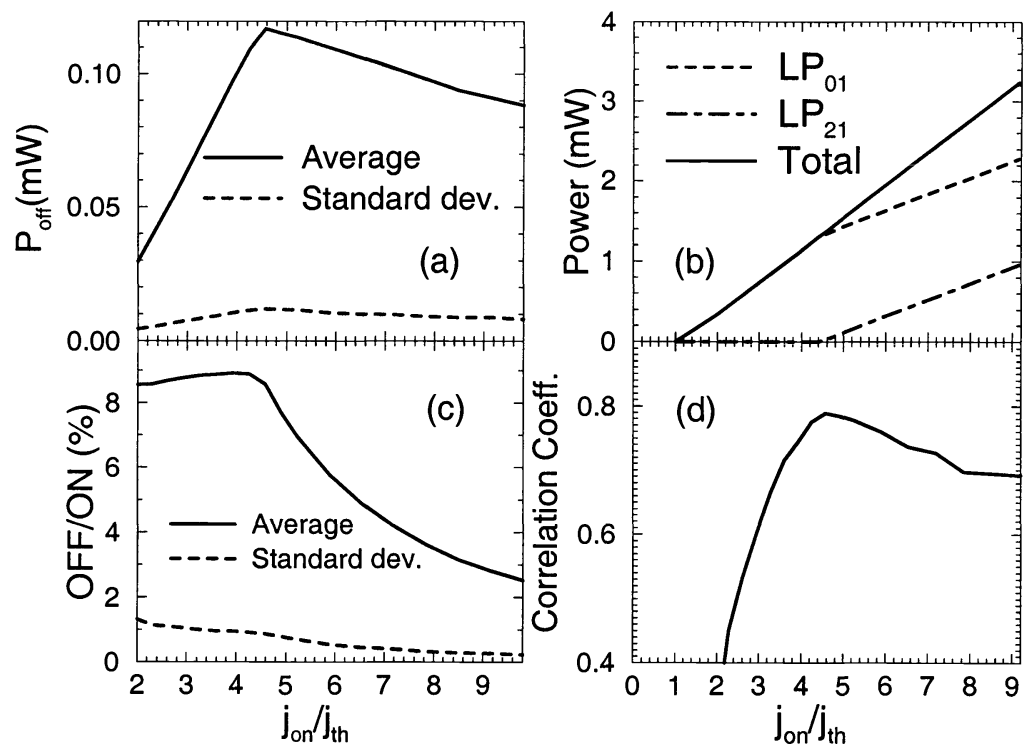

Figure 4. (a) Averaged maximum power of the secondary pulsation, $\left\langle P_{o f f}\right\rangle$ (solid line) and standard deviation of $P_{o f f}$ (dashed line) vs $j_{o n}$. (b) Multimode Light-Current characteristics. (c) Averaged $O F F / O N,\langle O F F / O N\rangle$ (solid line) and standard deviation of $O F F / O N$ (dashed line) vs $j_{o n}$. (d) Correlation coefficient of the relation $P_{o f f}$ vs $T_{o f f}$. Averages have been performed over 1000 modulation periods. 
The dependence of the secondary pulsations on the transverse mode behaviour of the VCSEL is now analysed by considering the averaged maximum power of secondary pulsations, $\left\langle P_{o f f}\right\rangle$ as a function of $j_{o n}$. This is shown in Fig. 4(a). For $j_{o n}<4.5 j_{t h}$, the VCSEL operates in the $L P_{01}$ mode in such a way that $\left\langle P_{o f f}\right\rangle$ increases with $j_{o n}$. In this regime an increase of $j_{\text {on }}$ leads to a bigger carrier density gradient due to SHB. This occurs in regions where the $L P_{01}$ mode has most of the power and then, the contribution of the diffusion current makes $P_{\text {off }}$ to increase with $j_{o n}$.

The situation changes when $j_{o n}>4.5 j_{t h}$ because the laser becomes multimode. Now, secondary pulsations in the $L P_{01}$ mode still appear but $\left\langle P_{o f f}\right\rangle$ slightly decreases when increasing $j_{o n}$. In this situation, several modes $\left(L P_{01}\right.$ and $\left.L P_{21}\right)$ are simultaneously lasing before the VCSEL is turned-off, as it can be seen from Fig. 4(b). The contribution of $L P_{21}$ modes causes the hole in the carrier density to be shallower in the region where the $L P_{01}$ mode has appreciable power. Then the radial component of the current density, (proportional to the carrier density gradient) decreases when $j_{o n}$ increases. In this way the additional current due to diffusion effects decreases with $j_{o n}$, and hence $\left\langle P_{o f f}\right\rangle$ slightly decreases when increasing $j_{o n}$. The quantity that characterises the strength of turn-off transients is the $O F F / O N$ ratio, defined as $P_{\text {off }}$, divided by the averaged total power in the on-steady state, $\left\langle P_{o n}\right\rangle$. In Fig. 4(c), it can be seen that the averaged $O F F / O N,\langle O F F / O N\rangle=\left\langle P_{o f f}\right\rangle /\left\langle P_{o n}\right\rangle$, slightly increases with $j_{o n}$, while the laser is single mode. That indicates that $\left\langle P_{\text {off }}\right\rangle$ increases slightly quicker than $\left\langle P_{o n}\right\rangle$ with $j_{o n}$. However, for $j_{o n}>4.5 \mathrm{kA} / \mathrm{cm}^{2}$, the VCSEL enters in a multimode regime and $\langle O F F / O N\rangle$ decreases with $j_{o n}$ [see Fig. 4(c)] because $\left\langle P_{o f f}\right\rangle$ also decreases [see Fig. 4(a)] and $\left\langle P_{o n}\right\rangle$ increases linearly with $j_{o n}$. Then $\langle O F F / O N\rangle$ decreases with $j_{o n}$ in the multimode regime and has a maximum for $j_{\text {on }}=4.5 \mathrm{kA} / \mathrm{cm}^{2}$, current at which the laser becomes multimode. The strength of the fluctuations is also shown in Figs. 4(a,c), where the standard deviation of $P_{\text {off }}$ and $O F F / O N, \sigma_{P o f f}$ and $\sigma_{O F F / O N}$, respectively, have been plotted with dashed lines. The qualitative behaviour of $\sigma_{P o f f}$ is very similar to the one of $\left\langle P_{o f f}\right\rangle$, having also a maximum at $j_{o n}=4.5 \mathrm{kA} / \mathrm{cm}^{2}$. However, $\sigma_{O F F / O N}$ decreases when $j_{o n}$ increases. This occurs since $\sigma_{O F F / O N}=\sigma_{P o f f} /\left\langle P_{o n}\right\rangle$ and changes in $\sigma_{P o f f}$ are small while $\left\langle P_{o n}\right\rangle$ increases quickly with $j_{o n}$. Correlation coefficient between $P_{o f f}$ and $T_{\text {off }}$ has the same qualitative behaviour than $O F F / O N$ and $P_{\text {off }}$, as it can be seen in Fig. 4(d). Again, the maximum correlation is obtained for the current at which the VCSEL becomes multimode. The above described results show that turn-off transients behaviour depends on the transverse mode character of the VCSEL.

\section{SPATIO-TEMPORAL DESCRIPTION OF VCSEL DYNAMICS}

A more complete description of the VCSEL dynamics to the one presented in the first part of this paper, focuses on the allowed transition among the magnetic sublevels of a quantum well (QW). Our model constitutes a natural generalization of the spin flip model (SFM) ${ }_{1}^{15}$ extensively used in the literature to describe polarization dynamics in VCSELs. The original SFM is extended to account for transverse effects and for a realistic frequencydependent susceptibility of the active material, which is assumed to be composed by one or several quantum wells (QW). The model describes the lateral dependence of the slowly-varying amplitudes (SVA) of the electric field expressed in their circular components. Since the circular basis is the natural representation of the optical transitions in QW-VCSELs, it is also natural to split the carrier densities into spin-up and spin-down carrier reservoirs. The evolution of the optical field and carrier variables is governed by ${ }^{16}$

$$
\begin{aligned}
\partial_{t} E_{ \pm}\left(\vec{r}_{\perp} ; t\right)= & -\kappa E_{ \pm}+i \hat{\mathcal{L}} E_{ \pm}+i \frac{a \Gamma}{2} P_{ \pm}\left(\vec{r}_{\perp} ; t\right) \\
& -\left(\gamma_{a}+i \gamma_{p}\right) E_{\mp}+\sqrt{\beta A D_{ \pm}} \xi_{ \pm}\left(\vec{r}_{\perp} ; t\right), \\
\partial_{t} D_{ \pm}\left(\vec{r}_{\perp} ; t\right)= & \frac{\mu(t)}{2} C\left(\vec{r}_{\perp}\right)-A D_{ \pm} \mp \gamma_{j}\left(D_{+}-D_{-}\right) \\
& +\mathcal{D} \nabla_{\perp}^{2} D_{ \pm}+\frac{a}{2 i}\left(P_{ \pm} E_{ \pm}^{*}-P_{ \pm}^{*} E_{ \pm}\right) .
\end{aligned}
$$

The SVA components of the electric field, $E_{ \pm}$, and the material polarization, $P_{ \pm}$, are expressed with respect to the longitudinal mode resonance frequency $\Omega$, that corresponds to a flat lateral distribution in refractive index. The carrier distributions $D_{ \pm}$are normalized to the transparency carrier density $N_{t}$. The cavity decay rate is $\kappa$, 


\begin{tabular}{|ccrc|}
\hline Symbol & Meaning & Value & Dimensions \\
\hline \hline$a \chi_{0}$ & effective gain constant & $1.3 \times 10^{4}$ & $\mathrm{~ns}^{-1}$ \\
$A_{0}$ & gain coefficient & $3 \times 10^{-16}$ & $\mathrm{~cm}^{2}$ \\
$\Gamma$ & longitudinal confinement factor & 0.045 & - \\
$\gamma$ & polarization decay rate & 10 & $\mathrm{ps}^{-1}$ \\
$n_{e}$ & background refractive index & 3.3 & - \\
$n_{g}$ & group refractive index & 3.5 & - \\
$\lambda$ & free-space wavelength & 0.85 & $\mu \mathrm{m}$ \\
$\sigma$ & bandgap shrinkage & 0.2 & - \\
$b$ & empty band contribution to & $2 \times 10^{4}$ & - \\
$\kappa$ & cavity losses & 300 & $\mathrm{~ns}^{-1}$ \\
$\gamma_{a}$ & linear dichroism & 0.5 & $\mathrm{~ns}^{-1}$ \\
$\gamma_{p}$ & linear birefringence & 15 & $\mathrm{~ns}^{-1}$ \\
$A$ & non-radiative recombination rate & 1.0 & $\mathrm{~ns}^{-1}$ \\
$N_{t}$ & transparent carrier density & $10^{18}$ & $\mathrm{~cm}^{-3}$ \\
$\gamma_{J}$ & spin flip rate & 50 & $\mathrm{~ns}^{-1}$ \\
$\mathcal{D}$ & bimolecular diffusion & 0.5 & $\mu \mathrm{m}^{2} \mathrm{~ns}^{-1}$ \\
$\beta$ & spontaneous emission factor & $2 \times 10^{-5}$ & \\
\hline
\end{tabular}

Table 1. Device and material parameters

while $\gamma_{a}$ and $\gamma_{p}$ stand for the amplitude and phase anisotropies. The lateral variation in passive refractive index is described in Eq. (5) through the waveguide operator that reads

$$
\hat{\mathcal{L}} E_{ \pm}=\frac{c^{2}}{2 \Omega n_{e} n_{g}}\left[\nabla_{\perp}^{2}+\left(\frac{\Omega}{c}\right)^{2} 2 n_{e} \Delta n\left(\vec{r}_{\perp} ; \Omega\right)\right] E_{ \pm},
$$

since we have assumed weak guidance, i.e. $\Delta n\left(\vec{r}_{\perp} ; \Omega\right)<<n_{e} . \Delta n$ is the excess refractive index contribution that arises from thermal lensing and from the oxide layer in the case of oxided VCSELs. The constitutive relationships, in Fourier domain, for the SVA of the electric field and material polarization read

$$
P_{ \pm}\left(\vec{r}_{\perp} ; \nu\right)=\chi_{ \pm}\left(\Omega+\nu, D_{+}, D_{-}\right) E_{ \pm}\left(\vec{r}_{\perp} ; \nu\right),
$$

that can be approximated in time domain by ${ }^{16}$

$$
P_{ \pm}\left(\vec{r}_{\perp} ; t\right) \approx \chi_{ \pm}\left(\Omega+i \frac{\partial_{t} E_{ \pm}\left(\vec{r}_{\perp} ; t\right)}{E_{ \pm}\left(\vec{r}_{\perp} ; t\right)}, D_{+}, D_{-}\right) E_{ \pm}\left(\vec{r}_{\perp} ; t\right)
$$

The effective gain constant is $a \equiv \Omega /\left(n_{e} n_{g}\right), \Gamma$ is the longitudinal confinement factor, while $\chi_{ \pm}$stands for a generic susceptibility function. In this work we use an analytical approximation to the optical susceptibility components, as those derived in Ref. [17], that read

$$
\chi_{ \pm}\left(\Omega+\nu, D_{+}, D_{-}\right)=-\chi_{0}\left[\ln \left(1-\frac{2 D_{ \pm}}{u+i}\right)+\ln \left(1-\frac{D_{+}+D_{-}}{u+i}\right)-\ln \left(1-\frac{b}{u+i}\right)\right]
$$

The first term on the right-hand side represents the contribution of the electrons, the second that of the holes, and the third represents the susceptibility of the system when no carriers are excited. The frequency dependence is introduced through

$$
u=\Delta+\frac{\nu}{\gamma}+\sigma\left(D_{-}+D_{+}\right)^{1 / 3}
$$

in which $\Delta=\left(\Omega-\omega_{g}\right) / \gamma$ measures the normalized detuning of the longitudinal mode resonance with respect to the nominal bandgap, and $\sigma\left(D_{-}+D_{+}\right)^{1 / 3}$ phenomenologically describes bandgap renormalization due to Coulomb interaction between electrons and holes. The rest of parameters are described in Table 1. 
We consider that the lateral distribution of current density is fixed by the structure of the device, so we express $J\left(\vec{r}_{\perp} ; t\right)=e W N_{t} C\left(\vec{r}_{\perp}\right) \mu(t)$, where $C\left(\vec{r}_{\perp}\right)$ is the current shape and $\mu(t)$ its time dependence. Hence, the total injected current reads

$$
I(t)=\mu(t) e N_{t} W \iint_{-\infty}^{\infty} C\left(\vec{r}_{\perp}\right) d^{2} \vec{r}_{\perp},
$$

$N_{t}$ being the (total) transparent carrier density, $e$ the absolute charge of the electron, and $W$ the total width of the QW active region. The parameters in Eq. (6) are: $A$ is the spontaneous recombination rate, $\gamma_{j}$ is the spin flip rate, and $\mathcal{D}$ is the carrier diffusion. Finally, we have phenomenologically added stochastic Langevin terms with zero mean and uncorrelated in both space, time and polarization to the equation for each electric field in order to model spontaneous emission processes.

\subsection{Numerical Simulations}

In this section we numerically implement the spatio-temporal model to study the turn-off transients of gainguided VCSEL. We apply an injection current that consists in a periodic square signal going from below to above threshold. We begin our discussion considering a situation where the fundamental transverse mode is stable even for injection currents far from threshold. In a second step, we analyze the role of the carrier-induced refractive index by varying the thermal lensing strength. Finally, we discuss the effect of a ring-shaped current distribution and the impact of multimode operation on the turn-off transients.

For the sake of simplicity, we assume that the radial distribution of injection current in the QW layer is approximated by the functional form

$$
C(\rho)=e^{-\rho^{6}} e^{n \rho^{2}},
$$

with $\rho=2 r / \phi_{c}$ and $\phi_{c}$ being the current diameter. The current distribution is supergaussian when $n=0$ corresponding to a disc-shaped electrical contact, while it becomes more ring-shaped as $n>0$ is increased. On the other hand, the distribution of passive refractive index is approximated by a truncated parabolic waveguide in order to mimic a thermal lens

$$
\Delta n(R)=\left\{\begin{array}{ccc}
\Delta n_{t l}\left[1-R^{2}\right] & \text { if } & R<1 \\
0 & \text { if } & R \geq 1
\end{array},\right.
$$

with $R \equiv 2 r / \phi_{g}, \phi_{g}$ being the thermal lens diameter. $\Delta n_{t l}$ controls the change in refractive index, that is measured with respect to the substrate value.

\subsubsection{Fundamental transverse mode operation}

We begin our discussion considering a situation where the waveguide operator $\hat{\mathcal{L}}$ admits only one guided solution -fundamental transverse mode-. Single-mode operation is obtained when small diameters and moderate thermal lensing strength are considered.

As a starting point, we focus on a VCSEL with a disc-shaped electric contact being modulated by a square signal going from threshold to 4 times threshold. The modulation period is large enough, $T=10 \mathrm{~ns}$, to allow the system reach CW operation. Following the evolution of the total intensity [Fig. 5(a)] during the current turn-off, we can see the appearance of a secondary optical pulse, even when the bias current is set at threshold or slightly below threshold. We explicitly define a secondary pulsation as the first optical peak that follows the current turnoff. These pulsations are characterized by two variables, the $O F F / O N$ ratio and the $T_{\text {off }}$ time. These pulsations can not be explained in terms of the typical relaxation oscillations that appears in gain-switched sc lasers. In contrast to relaxation oscillations, secondary pulsations are explained as a diffusive spatial effect. When the laser switches-on, the spatial-hole burning effect causes a hole in the center of the carrier distributions. When the current is turned-off, and under appropriate conditions, the hole in the carrier distribution is filled by diffusion processes, providing extra gain during a short period that finally produces an optical pulse. When comparing with previous results for similar mode profiles, we find that the modal expansion leads to an underestimate of the $O F F / O N$ of the secondary pulsations. A possible explanation of this phenomenon is given below. 


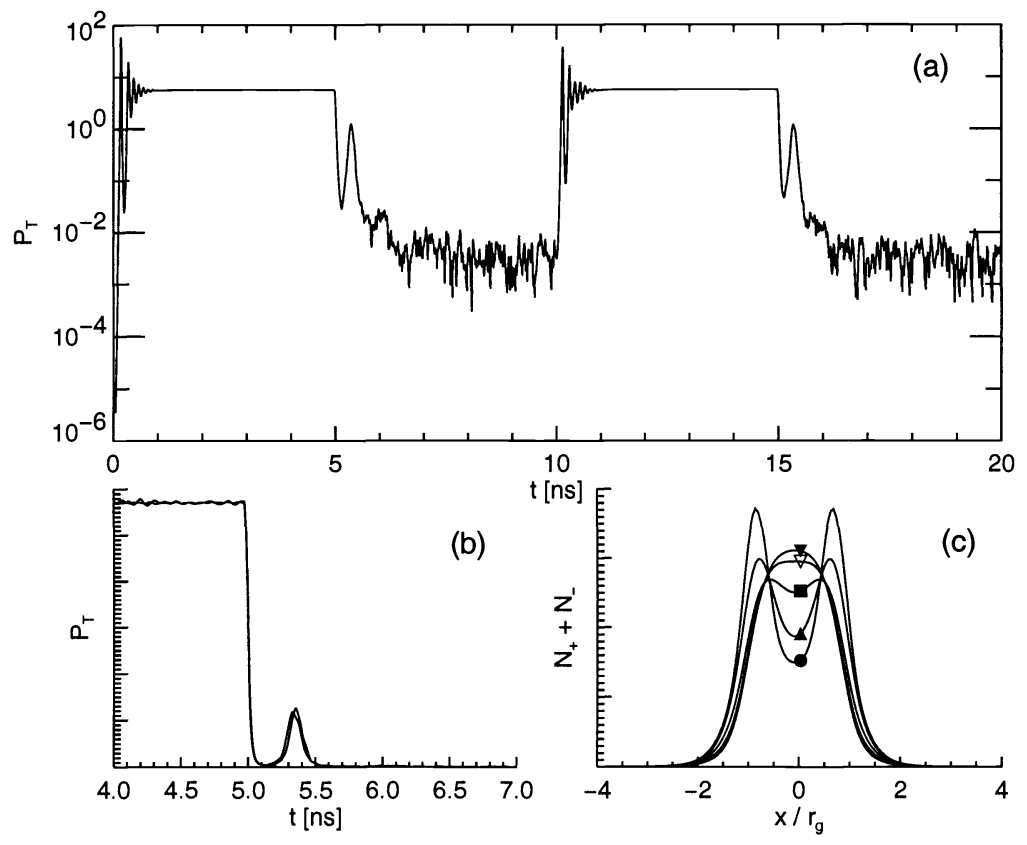

Figure 5. Numerical simulation of the spatio-temporal dynamics: (a) time trace of the total intensity in logscale, (b) zoom in linear scale, and (c) cross-sections of the total carrier distribution at $t=4 \mathrm{~ns}$ (solid circle), $t=5.25 \mathrm{~ns}$ (solid triangle), $t=5.83 \mathrm{~ns}$ (square), $t=6.4 \mathrm{~ns}$ (inverted triangle), and $t=7 \mathrm{~ns}$ (solid inverted triangle). Secondary pulsations with $O F F / O N \sim 21 \%$ and $T_{o f f} \sim 0.36 \mathrm{~ns}$. The optical profiles are shown in Fig. 6(c). Parameters: supergaussian current profile, $\phi_{c}=\phi_{g}=6 \mu \mathrm{m}, \mu_{\text {bias }}=\mu_{t h}, \mu_{o n}=4 \mu_{t h}, \Delta n_{t l}=3 \times 10^{-3}$, $\Delta=1.0$, and $\mu_{t h}$ represents the threshold current. The remaining parameters are given in Table 1.
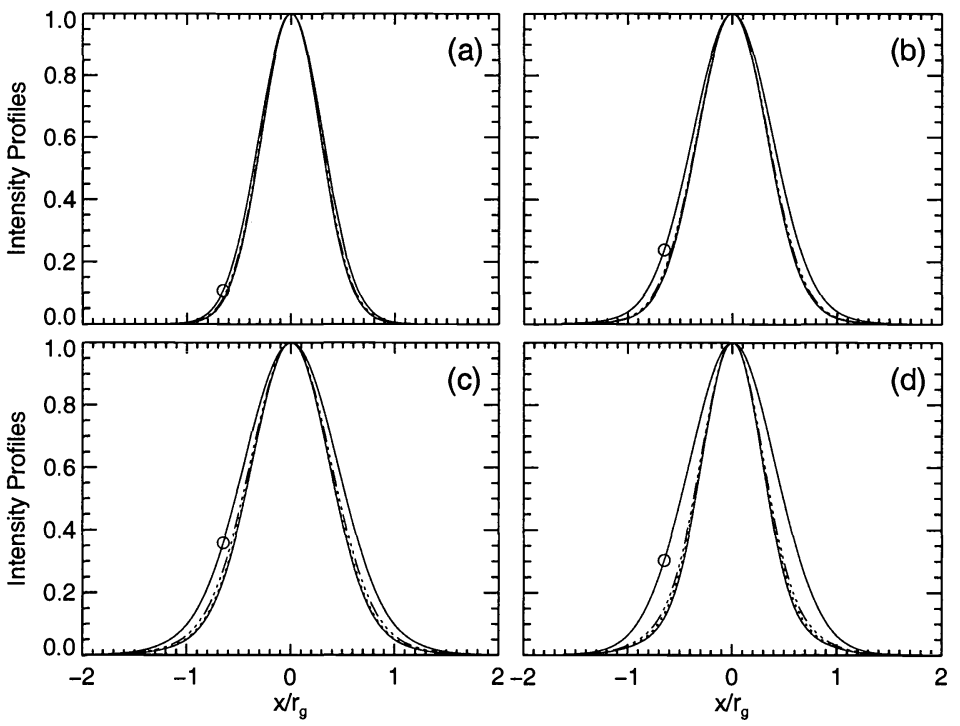

Figure 6. Optical profiles computed from the numerical integration of the spatio-temporal dynamics, at different time stages: beginning of the laser onset (solid lines + circle), under continuous wave operation (solid lines), first optical valley (dashed-dotted lines) and first peak of secondary pulsations (dotted lines). The mode shrinkage is (a) $\sim 7 \%$ for $\Delta n_{t l}=10^{-2}$, (b) $\sim 12 \%$ for $\Delta n_{t l}=5 \times 10^{-3}$, (c) $\sim 17 \%$ for $\Delta n_{t l}=3 \times 10^{-3}$, and (d) $\sim 30 \%$ for $\Delta n_{t l}=9 \times 10^{-4}$. 


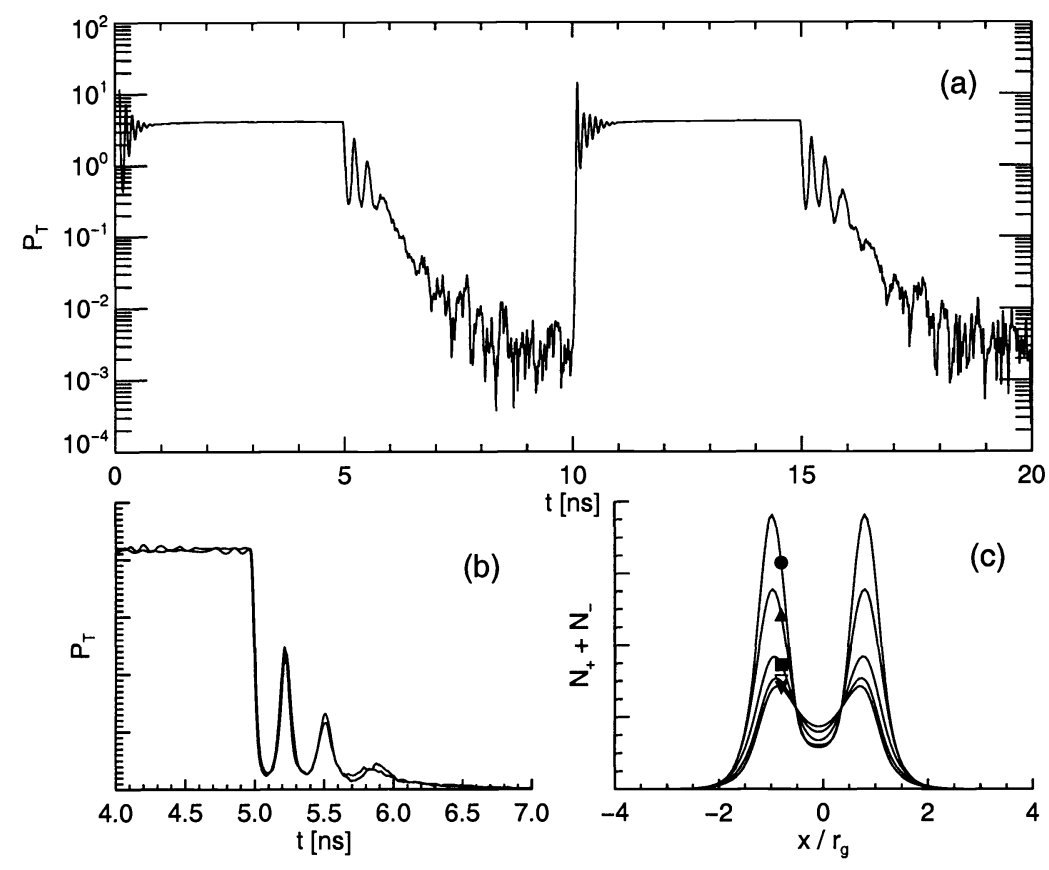

Figure 7. Numerical simulation of the spatio-temporal dynamics for a ring-shaped electrical contact with $(n=2)$. Secondary pulsations with $O F F / O N \approx 57 \%$ and $T_{\text {off }} \approx 0.2 \mathrm{~ns}$. Parameters: $\phi_{c}=\phi_{g}=6 \mu \mathrm{m}$, $\mu_{\text {bias }}=\mu_{t h}, \mu_{o n}=4 \mu_{t h}, \Delta n_{t l}=3 \times 10^{-3}$, and $\Delta=1.0$. Carrier distribution is plotted at times given in Fig. 5 . The remaining parameters are given in Table 1.

In this model, the modal profiles and frequencies are influenced by the carrier density, and we investigate how robust are the modal profiles during the different stages of the switch-on and turn-off dynamics. In Fig. 6, we represent a cross-section of the optical profiles at different time stages. For large thermal lensing strength, the modal profiles and modal frequencies are quite robust against perturbations produced by the carrier-induced refractive index. In this limit, the modal expansion, presented in Sec. II, reasonably provides an accurate description of the turn-off dynamics. However, this is not the case when $\Delta n_{t l}$ becomes small and comparable to the carrier-induced contribution. In the latter, following the laser switch-on, we observe a considerable mode shrinkage due to SHB in the carrier distribution. The distribution of refraction index is larger in the center than in the carrier crowding radius. This induces a waveguide which provides an extra lateral confinement of the electric field that, in turn, is the responsible of the mode shrinkage. The primary effect of thermal lensing is that the lateral extension of the modal profiles increases when thermal lensing decreases, therefore leading to a reduction of the secondary pulsations when describing the system in terms of a modal expansion. On the other hand, when thermal lensing is weak, it has been already commented that spatial hole burning produces an extra lateral confinement of the field favoring the activation of secondary pulsations.

In order to illustrate the effect of a ring-shaped current profile, we return to the situation described in Fig. 5. For the moderate value of the carrier diffusion we use, $\mathcal{D}=0.5 \mu \mathrm{m}^{2} / \mathrm{ns}\left(L_{D} \approx 0.7 \mu \mathrm{m}\right)$, the 'off' steady-state displays a hole in the center of the carrier distribution. In contrast to previous examples, the carrier distribution develops a marked peak at the carrier crowding radius in order to provide sufficient gain for the Gaussian mode to lase. In general large carrier densities are required due to the small overlap of the Gaussian mode with the ring-shaped carrier distribution. In addition, the first-order transverse mode is unfavored since the waveguide is single-mode. This effect finally leads to carrier distributions with a strong gradient. When the current is turn-off, this large gradient produces a rapidly filling of the hole, providing small $T_{\text {off }}$ times and rather large $O F F / O N$ ratios [Fig. 7] when comparing with the disc-shaped electrical contact. 


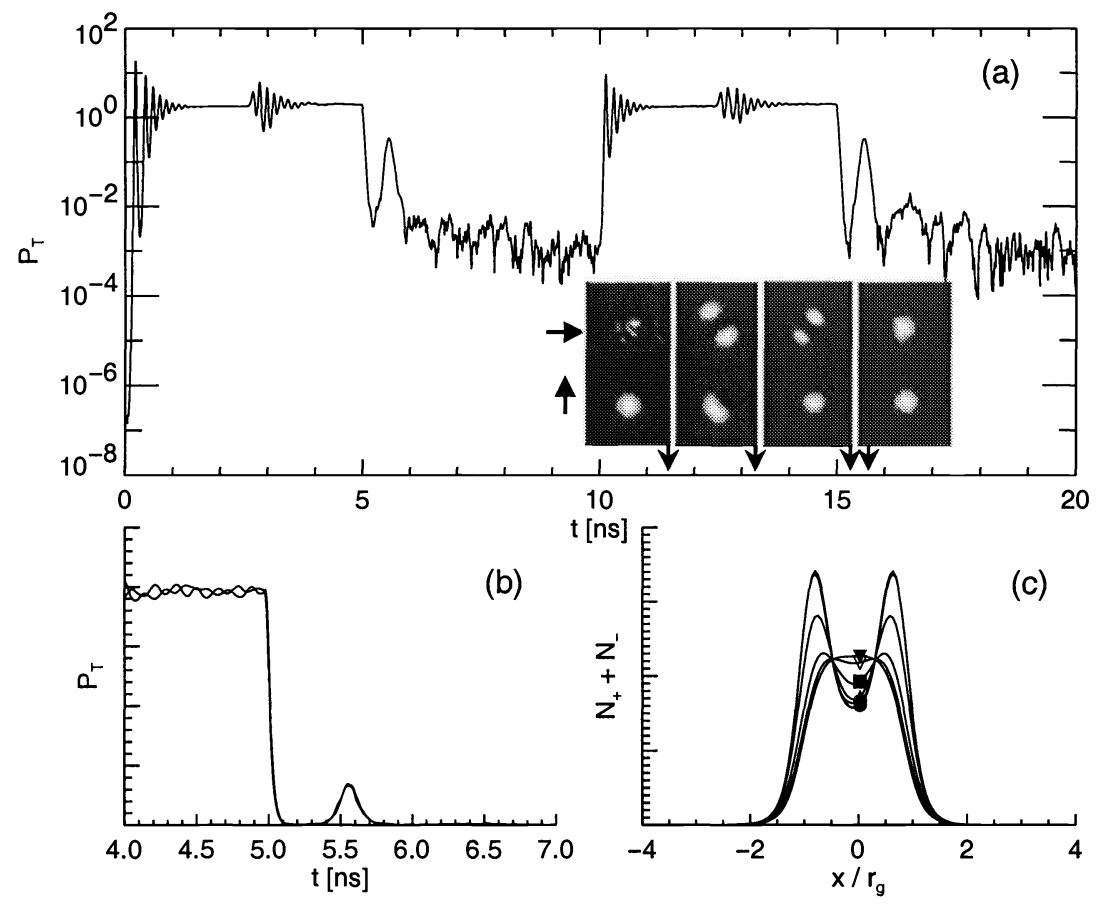

Figure 8. Numerical simulation of the spatio-temporal dynamics. Secondary pulsations in a multimode VCSEL, $O F F / O N \approx 15 \%$ and $T_{o f f} \approx 0.51 \mathrm{~ns}$. Parameters: supergaussian current profile, $\phi_{c}=\phi_{g}=8 \mu \mathrm{m}, \mu_{b i a s}=\mu_{t h}$, $\mu_{o n}=3 \mu_{t h}, \Delta n_{t l}=5 \times 10^{-3}$, and $\Delta=1.0$. Carrier distribution is plotted at times given in Fig. 5. The remaining parameters are given in Table 1.

\subsubsection{Role of higher order transverse modes}

As a final example, we consider a multimode VCSEL with larger diameter $\left(\phi_{g}=\phi_{c}=8 \mu \mathrm{m}\right)$ and moderate values for the thermal lensing $\left(\Delta n_{t l}=5 \times 10^{-3}\right)$. In Fig. 8 we show the results of the numerical simulations when the 'on' current is set to 3 times threshold. In panel (a) we represent the evolution of the total intensity (in log scale) and the near field images in both linear polarizations at different time stages. Following the laser switch-on, we observe operation in the Gaussian mode $L P_{01}$ in a vertical linear polarization. Due to the global increase in carrier density and due to the spatial hole burning in the carrier distribution, the first order transverse mode $L P_{11}$ can profit from the available material gain. The result is that $\sim 2.5 \mathrm{~ns}$ after the laser switch-on, the first order transverse mode starts lasing in the horizontal polarization, undergoing additional relaxation oscillations, and coexisting with the Gaussian mode until the current is turned-off. Following the current turn-off, we find that secondary pulsations take place in the fundamental transverse mode and both linear polarizations are active. A cross-section of the carrier distributions during the current turn-off is shown in panel (c). Even though the role of higher-order transverse modes is to suppress the magnitude of secondary pulsations, we can still observe an $O F F / O N \approx 15 \%$ in panel (b).

\section{CONCLUSIONS}

In summary, we have described the turn-off transients appearing in current modulated multi-transverse mode VCSELs. This description has been performed by using two different approaches. Results from the modal expansion indicate that fluctuations of the quantities that characterise the secondary pulsations, maximum power and time at which it appears, are important. A linear relation between these quantities has been found. We have shown that the strength of turn-off transients has a maximum for an injection current that corresponds to the current at which the VCSEL enters the multi-transverse mode operation. The use of a detailed spatio-temporal model has shown that, under certain conditions, the role of carrier-induced refractive index is to increase the strength of secondary pulsations with respect to the one obtained when describing the VCSEL in terms of modal 
expansion methods. Results obtained by using both models indicate that multi-transverse-mode operation helps to avoid turn-off transients, particularly in cases in which the VCSEL is driven well above threshold in order to achieve a required modulation rate or a sufficient optical output power.

\section{ACKNOWLEDGEMENTS}

Financial support has been provided by CICYT (Spain) Project TIC99-0645 and by EC Human Potential Research Training Network VISTA (HPRN CT-2000-00034).

\section{REFERENCES}

1. J. Jonsson, M. Ghisoni, S. Hatzikonstantinidou, A. Kullander-Sjoberg, A. Risberg, R. Stevens, K. Streubel, J. Sveijer, R. M. von Wurtemberg, "Reliable vertical-cavity components for multimode data communication", SPIE Proc., vol. 3946, pp. 144-151, 2000.

2. A. Valle, J. Sarma, and K. A. Shore, "Secondary pulsations driven by spatial-hole burning in modulated vertical-cavity surface-emitting lasers", J. Opt. Soc. Am., vol. B12, no. 9, pp. 1741-1746, 1995.

3. J. Tatum, D. Smith, J. Guenter, R. Johnson. "High speed characteristics of VCSELs", SPIE Proc., vol. 3004, pp. 151-159, 1997.

4. J. J. Morikuni, P. V. Mena, A. V. Harton, K. W. Wyatt, S. M. Kang. "Spatially independent VCSEL models for the simulation of diffusive turn-off transients", J. Lightwave Technol., vol. 17, pp. 95-101, 1999.

5. J. A. Lehman, and R. A. Morgan, "850 nm VCSELs for high speed data communications applications", in Vertical-cavity surface-emitting lasers: Technology and applications, Eds. J. Cheng, N. K. Dutta, (Gordon and Breach Sci. Publ., Amsterdam), Chapter 3, pp. 133, 2000.

6. M. I. Cohen, A. A. Allerman, K. D. Choquette, C. Jagadish, "Electrically steerable lasers using wide aperture VCSELs ", IEEE Photonics Technol. Lett., vol. 13, no. 6, pp. 544-546, 2001.

7. C. J. Chang-Hasnain, J. P. Harbison, G. Hasnain, A. C. von Lehmen, L. T. Florez, and N. G. Stoffel, "Dynamic, polarization and transverse mode characteristics of VCSELs", IEEE J. Quantum Electron., vol. 27, pp. 1402-1409, 1991.

8. D. Vakhshoori, J. D. Wynn, G. J. Zydzik, R. E. Leibenguth, M. T. Asom, K. Kojima, and R. A. Morgan, "Top-surface emitting lasers with $1.9 \mathrm{~V}$ threshold voltage and the effect of spatial hole burning on their transverse mode operation and efficiencies", Appl. Phys. Lett., vol. 62, no. 13, pp. 1448-1450, 1993.

9. A. Valle and L. Pesquera, "Turn-off transients in current-modulated multi-transverse-mode vertical-cavity surface-emitting lasers ," Appl. Phys. Lett., vol. 79, no. 24, pp. 3914-3916, 2001.

10. A. Valle, P. Rees, L. Pesquera, and K. A. Shore, "High-frequency beam steering in vertical-cavity surfaceemitting lasers: optical gain and waveguiding effects", J. Opt. Soc. Am., vol. B16, no. 11, pp. 2045-2054, 1999.

11. A. Valle, J. Sarma, and K. A. Shore, "Spatial hole burning effects on the dynamic of VCSELs," IEEE J. Quantum Electron., vol. 31, pp. 1423-1431, 1995.

12. A. Valle, "Selection and modulation of high-order transverse modes in vertical cavity surface emitting lasers", IEEE J. Quantum Electron., vol. 34, no. 10, pp. 1924-1932, 1998.

13. J. A. Vukusic, H. Martinsson, J. S. Gustavsson, A. Larsson, "Numerical optimization of the single fundamental mode output from a surface modified vertical-cavity surface-emitting laser ", IEEE J. Quantum Electron., vol. 37, no. 1, pp. 108-117, 2001.

14. S. Balle, P. Colet, and M. San Miguel, "Statistics for the transient response of single-mode semiconductor laser gain switching ", Phys. Rev. A, vol. 43, no. 1, pp. 498-506, 1991.

15. M. San Miguel, Q. Feng, and J. V. Moloney, "Light-polarization dynamics in surface-emitting semiconductor lasers," Phys. Rev. A, vol. 52, pp. 1728-1739, 1995.

16. J. Mulet and S. Balle, Accepted in IEEE J. Quantum Electron..

17. S. Balle, "Simple analytical approximations for the gain and refractive index spectra in quantum-well lasers," Phys. Rev. A, vol. 57, pp. 1304-1312, 1998. 